\title{
GENERALIZED FIBONACCI AND LUCAS SEQUENCES AND ROOTFINDING METHODS
}

\author{
JOSEPH B. MUSKAT
}

Dedicated to the memory of D. H. Lehmer

\begin{abstract}
Consider the sequences $\left\{u_{n}\right\}$ and $\left\{v_{n}\right\}$ generated by $u_{n+1}=$ $p u_{n}-q u_{n-1}$ and $v_{n+1}=p v_{n}-q v_{n-1}, n \geq 1$, where $u_{0}=0, u_{1}=1, v_{0}=$ $2, v_{1}=p$, with $p$ and $q$ real and nonzero. The Fibonacci sequence and the Lucas sequence are special cases of $\left\{u_{n}\right\}$ and $\left\{v_{n}\right\}$, respectively. Define $r_{n}=u_{n+d} / u_{n}, R_{n}=v_{n+d} / v_{n}$, where $d$ is a positive integer. McCabe and Phillips showed that for $d=1$, applying one step of Aitken acceleration to any appropriate triple of elements of $\left\{r_{n}\right\}$ yields another element of $\left\{r_{n}\right\}$. They also proved for $d=1$ that if a step of the Newton-Raphson method or the secant method is applied to elements of $\left\{r_{n}\right\}$ in solving the characteristic equation $x^{2}-p x+q=0$, then the result is an element of $\left\{r_{n}\right\}$.

The above results are obtained for $d>1$. It is shown that if any of the above methods is applied to elements of $\left\{R_{n}\right\}$, then the result is an element of $\left\{r_{n}\right\}$. The application of certain higher-order iterative procedures, such as Halley's method, to elements of $\left\{r_{n}\right\}$ and $\left\{R_{n}\right\}$ is also investigated.
\end{abstract}

Fibonacci and Lucas numbers appear repeatedly in the works of the father of computational number theory, D. H. Lehmer, who contributed also to numerical analysis, notably [5]. To his memory is dedicated this extension of results of McCabe and Phillips [6] and Jamieson [4] about applying iterative formulas for solving nonlinear equations to ratios of generalized Fibonacci numbers.

\section{INTRODUCTION}

Let $p$ and $q$ be real and nonzero. Define the generalized Fibonacci sequence

$$
u_{0}=0, \quad u_{1}=1, \quad u_{n+1}=p u_{n}-q u_{n-1}, \quad n \geq 1,
$$

and the generalized Lucas sequence

$$
v_{0}=2, \quad v_{1}=p, \quad v_{n+1}=p v_{n}-q v_{n-1}, \quad n \geq 1 .
$$

Let $d$ be a natural number. If $u_{n} \neq 0$, define the ratio

$$
r_{n}=u_{n+d} / u_{n} \text {. }
$$

If $v_{n} \neq 0$, define the ratio

$$
R_{n}=v_{n+d} / v_{n} .
$$

Received by the editor July 27, 1992 and, in revised form, October 13, 1992.

1991 Mathematics Subject Classification. Primary 11B39; Secondary 65H05. 
Related to the recurrence relation appearing in (1.1) and (1.2) is the characteristic equation

$$
x^{2}-p x+q=0
$$

If the equation has two real and unequal roots, then when $d=1$, the sequences of ratios $\left\{r_{n}\right\}$ and $\left\{R_{n}\right\}$ converge to the root of larger modulus. If there is a double root, then the sequences $\left\{r_{n}\right\}$ and $\left\{R_{n}\right\}$ converge to this root. McCabe and Phillips determined the condition for a generalized Fibonacci sequence to have no zero members; a necessary condition is that equation (1.5) have complex roots $([6$, p. 554$])$. Their analysis can be adapted readily to generalized Lucas numbers, by Lemma 3 below.

If $\alpha$ and $\beta$ are the roots of (1.5), then they satisfy ([3, equation (1.4)])

$$
\alpha+\beta=p, \quad \alpha \beta=q, \quad(\alpha-\beta)^{2}=(\alpha+\beta)^{2}-4 \alpha \beta=p^{2}-4 q .
$$

If $\alpha=\beta$, then

$$
2 \alpha=p, \quad \alpha^{2}=q=(p / 2)^{2}, \quad p^{2}-4 q=4 \alpha^{2}-4 \alpha^{2}=0 .
$$

Lemma 1 ([3, equations $(2.6),(2.7)])$. If $\alpha$ and $\beta$ are the distinct roots of (1.5) and $n \geq 0$, then

$$
u_{n}=\left(\alpha^{n}-\beta^{n}\right) /(\alpha-\beta) \text { and } v_{n}=\alpha^{n}+\beta^{n} .
$$

Lemma 2. If $\alpha$ is the double root of (1.5) and $n \geq 0$, then $u_{n}=n(p / 2)^{n-1}$ and $v_{n}=2(p / 2)^{n}$.

If $d \geq 1$, and the roots of (1.5) are real, then the sequences of ratios $\left\{r_{n}=\right.$ $\left.u_{n+d} / u_{n}\right\}$ and $\left\{R_{n}=v_{n+d} / v_{n}\right\}$ will converge to the $d$ th power of a root of (1.5). In other words, the sequences of ratios $\left\{r_{n}\right\}$ and $\left\{R_{n}\right\}$ converge to a root of

$$
x^{2}-\left(\alpha^{d}+\beta^{d}\right) x+(\alpha \beta)^{d}=x^{2}-v_{d} x+q^{d}=0,
$$

by Lemmas 1 and 2 and (1.6) and (1.7).

Define the Aitken transformation by

$$
A\left(x, x^{\prime}, x^{\prime \prime}\right)=\left(x x^{\prime \prime}-x^{\prime 2}\right) /\left(x-2 x^{\prime}+x^{\prime \prime}\right) \text {. }
$$

Define the secant transformation $S\left(x, x^{\prime}\right)$ for equation (1.8) by

$$
S\left(x, x^{\prime}\right)=\frac{x\left(x^{\prime 2}-v_{d} x^{\prime}+q^{d}\right)-x^{\prime}\left(x^{2}-v_{d} x+q^{d}\right)}{\left(x^{\prime 2}-v_{d} x^{\prime}+q^{d}\right)-\left(x^{2}-v_{d} x+q^{d}\right)}=\frac{x x^{\prime}-q^{d}}{x+x^{\prime}-v_{d}},
$$

and the Newton-Raphson transformation $N(x)$ for equation (1.8) by

$$
N(x)=x-\left(x^{2}-v_{d} x+q^{d}\right) /\left(2 x-v_{d}\right)=\left(x^{2}-q^{d}\right) /\left(2 x-v_{d}\right) .
$$

McCabe and Phillips proved that, if $d=1$, then

(i) $A\left(r_{n-t}, r_{n}, r_{n+t}\right)=r_{2 n}$ if $r_{2 n} \neq 0$,

(ii) $S\left(r_{n}, r_{m}\right)=r_{n+m}$ if $r_{n+m} \neq 0$,

(iii) $N\left(r_{n}\right)=r_{2 n}$ if $r_{2 n} \neq 0$.

It is now possible to state the extensions. As long as division by zero is avoided, then

(i) $A\left(r_{n-t}, r_{n}, r_{n+t}\right)=r_{2 n}, \quad A\left(R_{n-t}, R_{n}, R_{n+t}\right)=r_{2 n}$,

(ii) $S\left(r_{n}, r_{m}\right)=r_{n+m}, \quad S\left(R_{n}, R_{m}\right)=r_{n+m}$,

(iii) $N\left(r_{n}\right)=r_{2 n}, \quad N\left(R_{n}\right)=r_{2 n}$, 
for any natural number $d$. The idea of considering $d>1$ is due to Jamieson [4], who applied it only to the ordinary Fibonacci sequence.

The other extension is to apply the Halley transformation $H(x)$, which is a third-order refinement of the Newton-Raphson transformation:

$$
H\left(r_{n}\right)=r_{3 n}, \quad H\left(R_{n}\right)=R_{3 n} .
$$

Note that in the latter case the image is a ratio of generalized Lucas numbers. The Newton-Raphson and Halley transformations are two members of a certain infinite family of transformations; proofs applicable to the infinite family will be given.

Applying any of these transformations to elements of the sequence $\left\{R_{n}\right\}$, where (1.5) has a double root $\alpha$, gives rise to division by zero. In this situation $R_{n}=(p / 2)^{d}=\alpha^{d}$ for every $n \geq 1$; i.e., $R_{n}$ is the root of (1.8), by Lemma 2 and (1.7). In this case the ratios are constant, so the sequence is trivial. In the sequel the transformations will be applied to $R_{n}$ under the assumption that (1.5) has distinct roots.

Section 2 contains a list of elementary relationships about generalized Fibonacci and Lucas numbers. In $\S 3$ the Aitken transformation is studied. Section 4 is devoted to the secant transformation. Section 5 begins with the presentation of the Halley transformation. Then an infinite family of transformations, which includes those of Newton-Raphson and Halley, is investigated.

\section{Properties of generalized FibonacCi and LuCAS NUMbers}

For $n>0$ define $v_{-n}=\alpha^{-n}+\beta^{-n}$. Then by (1.6) and Lemma 1,

$$
q^{n} v_{-n}=(\alpha \beta)^{n} v_{-n}=\beta^{n}+\alpha^{n}=v_{n}
$$

Similarly, if equation (1.5) has distinct roots, define $u_{-n}=\left(\alpha^{-n}-\beta^{-n}\right) /(\alpha-\beta)$. Then by (1.6) and Lemma 1 ([3, equation (2.17)])

$$
q^{n} u_{-n}=(\alpha \beta)^{n} u_{-n}=\left(\beta^{n}-\alpha^{n}\right) /(\alpha-\beta)=-u_{n}
$$

Formula (2.2) is applicable also if equation (1.5) has a double root, for if $u_{-n}$ is defined by $-n(p / 2)^{-n-1}$, then $q^{n} u_{-n}=-n(p / 2)^{-n-1}(p / 2)^{2 n}=-n(p / 2)^{n-1}=$ $-u_{n}$.

It is easy to verify that the recurrence relations in (1.1) and (1.2) are valid also for negative subscripts.

Lemma 3 ([3, equation (4.10)]). If $n$ is an integer, then $u_{2 n}=u_{n} v_{n}$.

Lemma 4. If $n, m$, and $e$ are integers, then

(a) $u_{n+e} u_{n-e}-u_{n}^{2}=-q^{n-e} u_{e}^{2}$,

(b) $u_{n+e} u_{m}-u_{n} u_{m+e}=-q^{m} u_{e} u_{n-m}$,

(c) $u_{n+e} u_{m+e}-q^{e} u_{n} u_{m}=u_{e} u_{n+m+e}$,

(d) $u_{n+e}-q^{e} u_{n-e}=v_{n} u_{e}$,

(e) $u_{n+e}-v_{e} u_{n}=-q^{e} u_{n-e}$.

On the right side of statements (a)-(d) of the following lemma, there appears the factor $p^{2}-4 q$. If (1.5) has a double root, then $p^{2}-4 q=0$, by (1.7). It suffices to show in the case of a double root, accordingly, that the left side of each of these statements vanishes. 
Lemma 5. If $n, m$, and $e$ are integers, then

(a) $v_{n+e} v_{n-e}-v_{n}^{2}=q^{n-e}\left(p^{2}-4 q\right) u_{e}^{2}$,

(b) $v_{n+e} v_{m}-v_{n} v_{m+e}=q^{m}\left(p^{2}-4 q\right) u_{e} u_{n-m}$,

(c) $v_{n+e} v_{m+e}-q^{e} v_{n} v_{m}=\left(p^{2}-4 q\right) u_{e} u_{n+m+e}$,

(d) $v_{n+e}-q^{e} v_{n-e}=\left(p^{2}-4 q\right) u_{n} u_{e}$,

(e) $v_{n+e}-v_{e} v_{n}=-q^{e} v_{n-e}$.

Lemma 6. If $n, m$, and $e$ are integers, then $u_{n+e} v_{m}-u_{n} v_{m+e}=q^{m} u_{e} v_{n-m}$.

Lemma 7 ([3, equation (4.13)]). If $n$ is an integer, then $u_{n}\left(v_{n}^{2}-q^{n}\right)=u_{3 n}$.

\section{The Aitken transformation}

Theorem 1. Let $n>t \geq 0$ be integers, and assume that division by zero does not occur. Then (A) $A\left(r_{n-t}, r_{n}, r_{n+t}\right)=r_{2 n} ;$ (B) if equation (1.5) has distinct roots, then $A\left(R_{n-t}, R_{n}, R_{n+t}\right)=r_{2 n}$.

Proof. We prove only part (A). The proof of part (B) is similar. By (1.3) and (1.9),

$$
\begin{aligned}
A\left(r_{n-t}, r_{n}, r_{n+t}\right) & =\frac{r_{n-t} r_{n+t}-r_{n}^{2}}{r_{n-t}-2 r_{n}+r_{n+t}} \\
& =\frac{\left(u_{n-t+d} / u_{n-t}\right)\left(u_{n+t+d} / u_{n+t}\right)-\left(u_{n+d} / u_{n}\right)^{2}}{u_{n-t+d} / u_{n-t}-2 u_{n+d} / u_{n}+u_{n+t+d} / u_{n+t}} \\
& =\frac{u_{n-t+d} u_{n+t+d} u_{n}^{2}-u_{n-t} u_{n+t} u_{n+d}^{2}}{u_{n}\left[u_{n-t+d} u_{n} u_{n+t}-2 u_{n+d} u_{n-t} u_{n+t}+u_{n+t+d} u_{n-t} u_{n}\right]} \\
& =\frac{\left(u_{n-t+d} u_{n+t+d}-u_{n+d}^{2}\right) u_{n}^{2}-\left(u_{n-t} u_{n+t}-u_{n}^{2}\right) u_{n+d}^{2}}{u_{n}\left[\left(u_{n-t+d} u_{n}-u_{n+d} u_{n-t}\right) u_{n+t}-\left(u_{n+d} u_{n+t}-u_{n+t+d} u_{n}\right) u_{n-t}\right]} \\
& =\frac{-q^{n-t+d} u_{t}^{2} u_{n}^{2}+q^{n-t} u_{t}^{2} u_{n+d}^{2}}{u_{n} u_{d}\left(q^{n-t} u_{t} u_{n+t}-q^{n} u_{t} u_{n-t}\right)},
\end{aligned}
$$

by Lemmas $4($ a) and $4($ b),

$$
=\frac{u_{t}\left(u_{n+d}^{2}-q^{d} u_{n}^{2}\right)}{u_{n} u_{d}\left(u_{n+t}-q^{t} u_{n-t}\right)}=\frac{u_{t} u_{d} u_{2 n+d}}{u_{n} u_{d} v_{n} u_{t}}
$$

by Lemmas $4(\mathrm{c})$ and $4(\mathrm{~d})$,

$$
=u_{2 n+d} / u_{2 n}=r_{2 n}
$$

by Lemma 3 and then (1.3).

\section{THE SECANT TRANSFORMATION}

Theorem 2. Let $n$ and $m$ be positive integers, and assume that division by zero does not occur. Then (A) $S\left(r_{n}, r_{m}\right)=r_{n+m}$; (B) if equation (1.5) has distinct roots, then $S\left(R_{n}, R_{m}\right)=r_{n+m}$.

Proof. We prove only part (B). The proof of part (A) is similar. By (1.4) and (1.10),

$$
S_{d}\left(R_{n}, R_{m}\right)=\frac{R_{n} R_{m}-q^{d}}{R_{n}+R_{m}-v_{d}}=\frac{\left(v_{n+d} / v_{n}\right)\left(v_{m+d} / v_{m}\right)-q^{d}}{v_{n+d} / v_{n}+v_{m+d} / v_{m}-v_{d}}
$$




$$
=\frac{v_{n+d} v_{m+d}-q^{d} v_{n} v_{m}}{v_{n+d} v_{m}+v_{n}\left(v_{m+d}-v_{d} v_{m}\right)}=\frac{\left(p^{2}-4 q\right) u_{d} u_{n+m+d}}{v_{n+d} v_{m}-q^{d} v_{n} v_{m-d}},
$$

by Lemmas $5(\mathrm{c})$ and $5(\mathrm{e})$,

$$
=\frac{\left(p^{2}-4 q\right) u_{d} u_{n+m+d}}{\left(p^{2}-4 q\right) u_{d} u_{n+m}}=\frac{u_{n+m+d}}{u_{n+m}}=r_{n+m},
$$

by Lemma $5(\mathrm{c})$ and then (1.3).

\section{The Newton-Raphson and Halley transformations} 131])

The Halley transformation for the equation $f(x)=0$ is given by $([1, \mathrm{p}$.

$$
H(x)=x-f(x) /\left[f^{\prime}(x)-f(x) f^{\prime \prime}(x) / 2 f^{\prime}(x)\right] .
$$

Applying the Halley transformation to equation (1.8) yields

$$
\begin{aligned}
H(x) & =x-\frac{x^{2}-v_{d} x+q^{d}}{\left(2 x-v_{d}\right)-\left(x^{2}-v_{d} x+q^{d}\right) /\left(2 x-v_{d}\right)} \\
& =\frac{x^{3}-3 q^{d} x+v_{d} q^{d}}{3 x^{2}-3 v_{d} x+v_{d}^{2}-q^{d}} .
\end{aligned}
$$

An infinite family of transformations, which includes those of NewtonRaphson and Halley, will now be investigated. To this end, define the homogeneous polynomials in $y$ and $z$ by

$$
u_{d}^{h} q^{-f} T_{h, f, d}(y, z)=-\sum_{k=0}^{h}\left(\begin{array}{l}
h \\
k
\end{array}\right)(-y)^{k} z^{h-k} u_{d k-f} .
$$

Lemma 8. For $i=0,1,2, \ldots, h$ define

$$
E(i)=u_{d}^{i} q^{i t} \sum_{k=0}^{h-i}\left(\begin{array}{c}
h-i \\
k
\end{array}\right)\left(-u_{t}\right)^{k} u_{t+d}^{h-i-k} u_{d k-f-i t}
$$

Then $E(i)$ is independent of $i$.

Proof. It suffices to show that if $0 \leq i \leq h-1$, then $E(i)=E(i+1)$. By definition, $\left(\begin{array}{l}j \\ k\end{array}\right)=0$ if $k<0$ or $k>j$. Thus

$$
\begin{aligned}
E(i)= & u_{d}^{i} q^{i t} \sum_{k=0}^{h-i}\left[\left(\begin{array}{c}
h-i-1 \\
k
\end{array}\right)+\left(\begin{array}{c}
h-i-1 \\
k-1
\end{array}\right)\right]\left(-u_{t}\right)^{k} u_{t+d}^{h-i-k} u_{d k-f-i t} \\
= & u_{d}^{i} q^{i t} \sum_{k=0}^{h-i-1}\left(\begin{array}{c}
h-i-1 \\
k
\end{array}\right)\left(-u_{t}\right)^{k} u_{t+d}^{h-i-k} u_{d k-f-i t} \\
& +u_{d}^{i} q^{i t} \sum_{j=0}^{h-i-1}\left(\begin{array}{c}
h-i-1 \\
j
\end{array}\right)\left(-u_{t}\right)^{j+1} u_{t+d}^{h-i-j-1} u_{d j+d-f-i t} \\
= & u_{d}^{i} q^{i t} \sum_{k=0}^{h-i-1}\left(\begin{array}{c}
h-i-1 \\
k
\end{array}\right)\left(-u_{t}\right)^{k} u_{t+d}^{h-i-k-1}\left(u_{t+d} u_{d k-f-i t}-u_{t} u_{d k+d-f-i t}\right)
\end{aligned}
$$




$$
=u_{d}^{i+1} q^{(i+1) t} \sum_{k=0}^{h-i-1}\left(\begin{array}{c}
h-i-1 \\
k
\end{array}\right)\left(-u_{t}\right)^{k} u_{t+d}^{h-i-1-k} u_{d k-f-(i+1) t}
$$

by Lemma 4(b),

$$
=E(i+1) \text {. }
$$

Theorem 3. If $u_{d} \neq 0$, then $T_{h, f, d}\left(u_{t}, u_{t+d}\right)=u_{h t+f}$.

Proof. By Lemma 8,

$$
u_{d}^{h} q^{-f} T_{h, f, d}\left(u_{t}, u_{t+d}\right)=-E(0)=-E(h)=-u_{d}^{h} q^{h t} u_{-h t-f} .
$$

By (2.2),

$$
T_{h, f, d}\left(u_{t}, u_{t+d}\right)=u_{h t+f}
$$

Lemma 9. For $0 \leq i \leq h, i$ even, define

$$
F(i)=u_{d}^{i} q^{i t} \sum_{k=0}^{h-i}\left(\begin{array}{c}
h-i \\
k
\end{array}\right)\left(-v_{t}\right)^{k} v_{t+d}^{h-i-k} u_{d k-f-i t} .
$$

For $0<s \leq h, s$ odd, define

$$
G(s)=-u_{d}^{s} q^{s t} \sum_{k=0}^{h-s}\left(\begin{array}{c}
h-s \\
k
\end{array}\right)\left(-v_{t}\right)^{k} v_{t+d}^{h-s-k} v_{d k-f-s t} .
$$

Then $F(i)=G(i+1)$ if $i<h$, and $G(i+1)=\left(p^{2}-4 q\right) F(i+2)$ if $i<h-1$.

Proof. We have

$$
\begin{aligned}
F(i) & =u_{d}^{i} q^{i t} \sum_{k=0}^{h-i}\left[\left(\begin{array}{c}
h-i-1 \\
k
\end{array}\right)+\left(\begin{array}{c}
h-i-1 \\
k-1
\end{array}\right)\right]\left(-v_{t}\right)^{k} v_{t+d}^{h-i-k} u_{d k-f-i t} \\
& =u_{d}^{i} q^{i t} \sum_{k=0}^{h-i-1}\left(\begin{array}{c}
h-i-1 \\
k
\end{array}\right)\left(-v_{t}\right)^{k} v_{t+d}^{h-i-k-1}\left(v_{t+d} u_{d k-f-i t}-v_{t} u_{d k+d-f-i t}\right) \\
& =-u_{d}^{i+1} q^{(i+1) t} \sum_{k=0}^{h-i-1}\left(\begin{array}{c}
h-i-1 \\
k
\end{array}\right)\left(-v_{t}\right)^{k} v_{t+d}^{h-i-k-1} v_{d k-f-(i+1) t}
\end{aligned}
$$

by Lemma 6 ,

$$
=G(i+1) .
$$

Continuing,

$$
\begin{aligned}
G(i+1) & =-u_{d}^{i+1} q^{(i+1) t} \sum_{k=0}^{h-i-1}\left[\left(\begin{array}{c}
h-i-2 \\
k
\end{array}\right)+\left(\begin{array}{c}
h-i-2 \\
k-1
\end{array}\right)\right]\left(-v_{t}\right)^{k} v_{t+d}^{h-i-k-1} v_{d k-f-(i+1) t} \\
& =-u_{d}^{i+1} q^{(i+1) t} \sum_{k=0}^{h-i-2}\left(\begin{array}{c}
h-i-2 \\
k
\end{array}\right)\left(-v_{t}\right)^{k} v_{t+d}^{h-i-k-2}\left(v_{t+d} v_{d k-f-(i+1) t}-v_{t} v_{d k+d-f-(i+1) t}\right) \\
& =u_{d}^{i+2} q^{(i+2) t}\left(p^{2}-4 q\right) \sum_{k=0}^{h-i-2}\left(\begin{array}{c}
h-i-2 \\
k
\end{array}\right)\left(-v_{t}\right)^{k} v_{t+d}^{h-i-k-2} v_{d k-f-(i+2) t},
\end{aligned}
$$


by Lemma $5(\mathrm{~b})$,

$$
=\left(p^{2}-4 q\right) F(i+2) .
$$

Theorem 4. Assume $u_{d} \neq 0$. If $h$ is even, then

$$
T_{h, f, d}\left(v_{t}, v_{t+d}\right)=\left(p^{2}-4 q\right)^{h / 2} u_{h t+f} \text {. }
$$

If $h$ is odd, then

$$
T_{h, f, d}\left(v_{t}, v_{t+d}\right)=\left(p^{2}-4 q\right)^{(h-1) / 2} v_{h t+f} .
$$

Proof. Apply Lemma $9[h / 2]$ times:

If $h$ is even, then

$$
\begin{aligned}
u_{d}^{h} q^{-f} T_{h, f, d}\left(v_{t}, v_{t+d}\right) & =-F(0)=-\left(p^{2}-4 q\right) F(2)=-\left(p^{2}-4 q\right)^{2} F(4) \\
& =\cdots=-\left(p^{2}-4 q\right)^{h / 2} F(h)=-u_{d}^{h} q^{h t}\left(p^{2}-4 q\right)^{h / 2} u_{-h t-f} .
\end{aligned}
$$

By (2.2), $T_{h, f, d}\left(v_{t}, v_{t+d}\right)=\left(p^{2}-4 q\right)^{h / 2} u_{h t+f}$.

If $h$ is odd, then

$$
\begin{aligned}
u_{d}^{h} q^{-f} T_{h, f, d}\left(v_{t}, v_{t+d}\right) & =-F(0)=-\left(p^{2}-4 q\right) F(2) \\
& =\cdots=-\left(p^{2}-4 q\right)^{(h-1) / 2} F(h-1) \\
& =-\left(p^{2}-4 q\right)^{(h-1) / 2} G(h)=\left(p^{2}-4 q\right)^{(h-1) / 2} u_{d}^{h} q^{h t} v_{-h t-f}
\end{aligned}
$$

By $(2.1), T_{h, f, d}\left(v_{t}, v_{t+d}\right)=\left(p^{2}-4 q\right)^{(h-1) / 2} v_{h t+f}$.

Define

$$
g_{h}(z / y)=\frac{-q^{d} \sum_{k=0}^{h}\left(\begin{array}{l}
h \\
k
\end{array}\right)\left(\frac{z}{-y}\right)^{h-k} u_{d(k-1)}}{-\sum_{k=0}^{h}\left(\begin{array}{l}
h \\
k
\end{array}\right)\left(\frac{z}{-y}\right)^{h-k} u_{d k}} .
$$

Multiply the numerator and the denominator of the fraction by $u_{d}^{-h}(-y)^{h}$ :

$$
g_{h}(z / y)=\frac{-u_{d}^{-h} q^{d} \sum_{k=0}^{h}\left(\begin{array}{l}
h \\
k
\end{array}\right)(-y)^{k} z^{h-k} u_{d(k-1)}}{-u_{d}^{-h} \sum_{k=0}^{h}\left(\begin{array}{l}
h \\
k
\end{array}\right)(-y)^{k} z^{h-k} u_{d k}}=\frac{T_{h, d, d}(y, z)}{T_{h, 0, d}(y, z)} .
$$

The immediate consequences of Theorems 3 and 4 are:

Theorem 5. (a) Assume that $u_{d} \neq 0$ and $u_{h t} \neq 0$. Then $g_{h}\left(u_{t+d} / u_{t}\right)=u_{h t+d} / u_{h t}$.

(b) Assume that $u_{d} \neq 0, v_{t} \neq 0$, and $v_{h t} \neq 0$. Then

$$
g_{h}\left(v_{t+d} / v_{t}\right)= \begin{cases}u_{h t+d} / u_{h t}, & h \text { even, } \\ v_{h t+d} / v_{h t}, & h \text { odd } .\end{cases}
$$

Theorem 6. If $n$ is a positive integer, and division by zero does not occur, then $N\left(r_{n}\right)=N\left(R_{n}\right)=r_{2 n}$.

Proof. In view of Theorem 5, it suffices to show that $g_{2}(z / y)=N(z / y)$, where $N(x)$ is given by equation (1.11). By (5.3),

$$
g_{2}(z / y)=\frac{-q^{d}\left(z^{2} u_{-d}+y^{2} u_{d}\right)}{-\left(-2 y z u_{d}+y^{2} u_{2 d}\right)}=\frac{z^{2} u_{d}-q^{d} y^{2} u_{d}}{2 y z u_{d}-y^{2} u_{d} v_{d}},
$$


by (2.2) and Lemma 3 ,

$$
=\frac{(z / y)^{2}-q^{d}}{2 z / y-v_{d}}=N(z / y) .
$$

Theorem 7. If $n$ is a positive integer, and division by zero does not occur, then $H\left(r_{n}\right)=r_{3 n}$ and $H\left(R_{n}\right)=R_{3 n}$.

Proof. In view of Theorem 5, it suffices to show that $g_{3}(z / y)=H(z / y)$, where $H(x)$ is given by equation (5.1). By (5.3),

$$
\begin{aligned}
g_{3}(z / y) & =\frac{-q^{d}\left(z^{3} u_{-d}+3 y^{2} z u_{d}-y^{3} u_{2 d}\right)}{-\left(-3 y z^{2} u_{d}+3 y^{2} z u_{2 d}-y^{3} u_{3 d}\right)} \\
& =\frac{z^{3} u_{d}-3 y^{2} z q^{d} u_{d}+y^{3} q^{d} u_{d} v_{d}}{3 y z^{2} u_{d}-3 y^{2} z u_{d} v_{d}+y^{3} u_{d}\left(v_{d}^{2}-q^{d}\right)}
\end{aligned}
$$

by (2.2), Lemma 3 , and Lemma 7 ,

$$
=\frac{(z / y)^{3}-3 q^{d}(z / y)+q^{d} v_{d}}{3(z / y)^{2}-3(z / y) v_{d}+v_{d}^{2}-q^{d}}=H(z / y)
$$

Remark. Theorem 3, with $f=0$ and $d=1$, resembles a formula given by $\mathrm{H}$. Siebeck, cited in [2, p. 394].

I wish to acknowledge helpful suggestions from my colleagues Professors $\mathrm{H}$. Furstenberg and S. Shnider.

\section{BIBLIOGRAPHY}

1. W. Gander, On Halley's iteration method, Amer. Math. Monthly 92 (1985), 131-134.

2. L. E. Dickson, History of the theory of numbers, Vol. 1, Chelsea, New York, 1952.

3. A. F. Horadam, Basic properties of a certain generalized sequence of numbers, Fibonacci Quart. 3 (1965), 161-176.

4. M. J. Jamieson, Fibonacci numbers and Aitken sequences revisited, Amer. Math. Monthly 97 (1990), 829-831.

5. D. H. Lehmer, A machine method for solving polynomial equations, J. Assoc. Comput. Mach. 8 (1961), 151-162.

6. J. H. McCabe and G. M. Phillips, Aitken sequences and generalized Fibonacci numbers, Math. Comp. 45 (1985), 553-558.

Department of Mathematics and Computer Science, Bar-Ilan University, 52900 RAMAT-GAN, ISRAEL

E-mail address: muskat@bimacs.cs.biu.ac.il 